\title{
Saccharum Officinarum Leaf Extract as Corrosion Inhibitor of Copper Corrosion in Sulphuric Acid Solution: Experiments and Theoretical Calculations
}

\author{
Zhengyuan Gao $^{l}$, Pengfei Sun ${ }^{l}$, Lianteng Du ${ }^{l}$, Xiang Zhang ${ }^{1}$, Jialong Bai ${ }^{l}$, Haojie Xing ${ }^{l}$, \\ Yongbo Yan $^{2, *}$ \\ ${ }^{1}$ School of Mechatronics \& Vehicle Engineering, Chongqing Jiaotong University, Chongqing, \\ 400074,China; \\ ${ }^{2}$ School of Oil and Natural Gas Engineering, Southwest Petroleum University, Chengdu 610500 \\ *E-mail: 779265488@qq.com, yanyongbo0520@163.com
}

Received: 11 July 2021 / Accepted: 30 August 2021 / Published: 10 October 2021

\begin{abstract}
Saccharum officinarum leaf extract was obtained by the ethanol soaking method in this study. Fourier infrared spectroscopy experiments show that Saccharum officinarum leaf extract contains a mass of oxygen or nitrogen-containing functional groups. Electrochemical experiments show that Saccharum officinarum leaf extract can efficaciously suppression the corrosion of $\mathrm{Cu}$ at $\mathrm{H}_{2} \mathrm{SO}_{4}$. Polarization curve test shows that the $\eta$ of Saccharum officinarum leaf extract can reach more than 93\%, and it is a mixedtype corrosion inhibitor. The morphology test indicates that after Saccharum officinarum leaf extract is adsorbed onto the $\mathrm{Cu}$ interface, the corrosion rate of the corrosive medium on the copper surface is significantly reduced. Quantum chemical (QC) calculations and molecular dynamics (MD) simulations have shown that Saccharum officinarum leaf extract can efficaciously control the corrosion of $\mathrm{Cu}$ at sulfuric acid solution.
\end{abstract}

Keywords: Saccharum officinarum leaf extract; Corrosion inhibitor; $\mathrm{H}_{2} \mathrm{SO}_{4}$; Copper; Quantum chemical calculations

\section{$\underline{\text { FULL TEXT }}$}

(C) 2021 The Authors. Published by ESG (www.electrochemsci.org). This article is an open access article distributed under the terms and conditions of the Creative Commons Attribution license (http://creativecommons.org/licenses/by/4.0/). 